ate was oxidized to ${ }^{14} \mathrm{CO}_{2}$ and incorporated into lipids at these concentrations.

5. The inhibitory effects of fatty acids are explained in terms of their effects on the enzyme systems involved in oxidative phosphorylation.

It is a pleasure to thank Professor J. H. Quastel, F.R.S., for his continued interest in this work and to acknowledge the financial support of the National Cancer Institute of Canada. One of us (K. A.) was the holder of a Colombo Plan Fellowship under the Technical Assistance Programme by the Government of Canada, Department of Trade and Commerce, to the Republic of Pakistan.

\section{REFERENCES}

Ahmed, K. \& Scholefield, P. G. (1960). Nature, Lond., 186, 1046.

Ahmed, K. \& Scholefield, P. G. (1961). Biochem. J. 60, 432.

Borgstrom, B., Sudduth, H. C. \& Lehninger, A. L. (1955). J. biol. Chem. 215, 571 .

Calvin, M., Heidelberger, C., Reid, J. C., Tolbert, B. M. \& Yankwich, P. E. (1949). Isotopic Carbon, p. 317. New York: John Wiley and Sons Inc.

Chappell, J. B. \& Greville, G. D. (1958). J. biol. Chem. 182 , 813.

Creaser, E. H., de Leon, R. P. \& Scholefield, P. G. (1959). Cancer Res. 19, 705.

Creaser, E. H. \& Scholefield, P. G. (1960). Cancer Res. 20, 257.

Cross, R. J., Taggart, J. V., Covo, G. A. \& Green, D. E. (1949). J. biol. Chem. 177, 655.
Dianzani, M. U. (1954). Biochim. biophys. acta, 14, 514.

Edson, N. L. (1935). Biochem. J. 29, 2082.

Fiske, C. H. \& Subbarow, Y. (1929). J. biol. Chem. 81, 629.

Hogeboom, G. H., Schneider, W. C. \& Pallade, G. E. (1948). J. biol. Chem. 172, 619.

Hunter, F. E. \& Ford, L. (1955). J. biol. Chem. 216, 357.

Judah, J. D. \& Rees, K. R. (1953). Biochem. J. 55, 664.

Kaplan, N. O. \& Greenberg, D. M. (1944). J. biol. Chem. 156, 511.

Lehninger, A. L. (1945). J. biol. Chem. 157, 363.

Lehninger, A. L. (1951). In Phosphorus Metabolism, vol. 1, p. 350. Ed. by McElroy, W. D. \& Glass, B. Baltimore, The Johns Hopkins Press.

Lehninger, A. L. (1959). J. biol. Chem. 234, 2465.

Lehninger, A. L. \& Remmert, L. F. (1959). J. biol. Chem. 234, 2459.

Lehninger, A. L., ul Hassan, M. \& Sudduth, H. C. (1954). J. biol. Chem. 210, 910.

Lynen, F. (1955). Annu. Rev. Biochem. 24, 653.

Polis, B. D. \& Schmukler, H. W. (1957). J. biol. Chem. 227, 419.

Pressman, B. C. \& Lardy, H. A. (1956). Biochim. biophys. acta, 21, 458.

Pullman, M. E. \& Racker, E. (1956). Science, 123, 1105.

Quastel, J. H. \& Wheatley, A. H. M. (1933). Biochem. J. 27, 1756.

Scholefield, P. G. (1956). Canad. J. Biochem. Physiol. 34, 1211, 1229.

Scholefield, P. G. (1958). Cancer Res. 18, 1026.

Sinclair, R. G. \& Dolan, M. (1942). J. biol. Chem. 142 , 659.

Biochem. J. (1961) 81, 45

\title{
Studies on Fatty Acid Oxidation
}

\section{THE EFFECTS OF FATTY ACIDS ON METABOLISM OF RAT-BRAIN CORTEX IN VITRO}

\author{
By K. AHMED* AND P. G. SCHOLEFIELD $\dagger$ \\ McGill-Montreal General Hospital Research Institute, 3619 University Street, Montreal, P.Q., Canada
}

(Received 3 November 1960)

In the preceding paper (Ahmed \& Scholefield, 1961 ) it was shown that fatty acids uncouple oxidation from phosphorylation in rat liver. The present studies were undertaken to survey the effects of fatty acids on various aspects of brain metabolism in vitro. These studies were of interest in view of the observation by Samson \& Dahl (1955) that injection of short-chain fatty acids produces narcotic-like effects in normal animals.

It was previously demonstrated that the rapid exchange reaction between adenosine triphosphate

* Colombo Plan Fellow.

$\dagger$ Research Associate of the National Cancer Institute of Canada. and ${ }^{32} \mathrm{P}$-labelled phosphate brought about by ratliver mitochondria is a significant factor in the labelling of adenosine triphosphate and other organic phosphates in rat-liver slices (Ahmed \& Scholefield, 1960). In the present paper the conditions necessary for measurement of the rate of the adenosine triphosphate-inorganic $\left[{ }^{32} \mathrm{P}\right]$ phosphate (ATP- ${ }^{32} \mathrm{P}_{i}$ ) exchange reaction in rat-brain mitochondria have been examined. Findlay, Rossiter \& Strickland (1953) observed that glucose and oxygen were essential for the incorporation of ${ }^{32} \mathrm{P}$ into the nucleic acid fraction of cat-brain slices. This labelling was markedly decreased when the members of the tricarboxylic acid cycle were used 
as substrates in place of glucose and also when conditions were made anaerobic. The effects of decanoate and dinitrophenol on the incorporation of ${ }^{32} \mathrm{P}$ into various fractions of rat-brain-cortex slices have now been investigated to determine the part played by synthesis of adenosine triphosphate in the labelling of the various organic phosphate fractions.

\section{MATERIALS AND METHODS}

Animals. Adult hooded rats of a local inbred strain, weighing approx. $200 \mathrm{~g}$., were used throughout this investigation.

Chemicals. All common chemicals were of reagent grade and were used without further purification. Adenosine triphosphate was obtained from Nutritional Biochemicals Corp., Cleveland, Ohio, U.S.A.; cytochrome $c$ from Wyeth Inc., Philadelphia, Pa., U.S.A.; and hexokinase from the Sigma Chemical Co., St Louis, Mo., U.S.A. Radioactive phosphate was purchased from Charles E. Frost and Co., Montreal, P.Q., Canada. It was purified as described by Ahmed \& Scholefield (1961). All the solutions and experimental media were adjusted to $\mathrm{pH} 7 \cdot 4$.

Tissue preparations. Rat-brain mitochondria were prepared according to Brody \& Bain (1952) by centrifuging rat-brain homogenates in $0.25 \mathrm{M}$-sucrose containing $1 \mathrm{mM}$ -

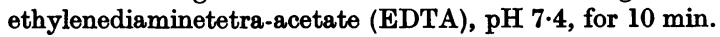
at $1200 \mathrm{~g}$ to remove nuclei and cell debris, and then at $10500 \mathrm{~g}$ for $10 \mathrm{~min}$. to obtain the mitochondrial pellet, which was washed once with $0.25 \mathrm{M}$-sucrose solution. All these manipulations were carried out at $2^{\circ}$.

Rat brains were removed and chilled in ice-cold KrebsRinger salts solution. Cortex slices were cut from cerebral hemispheres by means of a Stadie-Riggs tissue slicer (Arthur H. Thomas Co., Philadelphia, Pa., U.S.A.).

Incubation techniques. Oxygen uptake was measured with the conventional Warburg apparatus at $37^{\circ}$ in an atmosphere of $\mathrm{O}_{2}$ for slices and in air for mitochondria. For the measurement of aerobic glycolysis $\mathrm{O}_{2}+\mathrm{CO}_{2}(93: 7)$ was used and for anaerobic glycolysis $\mathrm{N}_{2}+\mathrm{CO}_{2}(93: 7)$ was used as gas phase.

The Krebs-Ringer medium used for incubation had the following composition: $\mathrm{NaCl}, 145 \mathrm{~mm}$; $\mathrm{KCl}, 5.8 \mathrm{~mm}$; $\mathrm{KH}_{2} \mathrm{PO}_{4}, 1.5 \mathrm{~mm} ; \mathrm{MgSO}_{4}, 1.5 \mathrm{~mm} ; \mathrm{CaCl}_{2}, 3.1 \mathrm{~mm}$ and sodium phosphate buffer, $\mathrm{pH} 7.4,10 \mathrm{~mm}$, the final $\mathrm{pH}$ being $\mathbf{7 \cdot 3}$. The volume of the incubation medium was $3 \mathrm{ml}$. in all cases. When aerobic or anaerobic glycolysis was measured, the phosphate buffer was replaced by $25 \mathrm{~mm}-\mathrm{NaHCO}_{3}$ to yield a final $\mathrm{pH}$ of 7.4. The media used for incubation of mitochondria are as described in the text.

Estimation of radioactivity. The methods used in assaying $\left[{ }^{82} \mathrm{P}\right]$ phosphate incorporation into slices and the $A T P-{ }^{82} \mathrm{P}_{i}$ exchange in mitochondria are as described in the preceding paper (Ahmed \& Scholefield, 1961). All counting was done to an accuracy of within $5 \%$.

Calculation of results. Most of the results are expressed in terms of $\mu \mathrm{m}$-moles of phosphate incorporated into the various fractions $/ 100 \mathrm{mg}$. wet wt. of tissue, calculated from the formula given in the preceding paper.

All experiments were performed at least twice and either typical or mean values are quoted.

\section{RESULTS}

\section{Effects of decanoate and other fatty acids on the respiratory activity of rat-brain-cortex slices}

Time course of respiration. Brain oxidizes fatty acids very slowly. When low concentrations of fatty acids are added to brain slices respiring in the presence of glucose, a slight stimulation of the rate of oxygen uptake is observed, but increase in the fatty acid concentration leads to the cessation of respiratory activity after an initial stimulatory phase. Thus $0.25 \mathrm{~mm}$-decanoate stimulated respiration throughout an incubation period of $120 \mathrm{~min}$. but when $0.5 \mathrm{~mm}$-decanoate was used there was an initial stimulation and after incubation for $90 \mathrm{~min}$. the rate of respiration fell off markedly (Fig. 1). A similar phenomenon was observed when 0.75 mM-decanoate was used but the decrease in the rate of oxygen uptake occurred after incubation for $60 \mathrm{~min}$. (Fig. 1). When $100 \mathrm{mM}-\mathrm{KCl}$ was added to the incubation medium, a stimulation of respiratory activity was observed both in the presence and in the absence of $0.5 \mathrm{~mm}$-decanoate, but the 'fall-off' of the respiratory activity in the former occurred after $60 \mathrm{~min}$. incubation compared with $90 \mathrm{~min}$. for brain cortex incubated with the standard amount of potassium $(7.3 \mathrm{~mm})$.

All the fatty acids tested, from heptanoate to myristate, produced inhibitory effects at higher concentrations. In Fig. 2 is shown the relationship between chain length of the fatty acids and the concentrations required to produce a 'fall-off' of

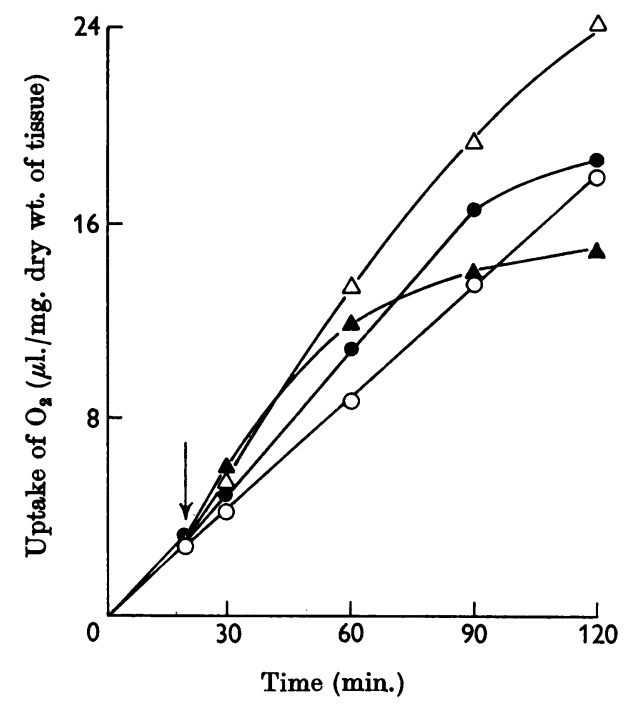

Fig. 1. Effects of $0.5 \mathrm{~mm}$-potassium decanoate and $100 \mathrm{~mm}$ $\mathrm{KCl}$ on the respiratory activity of rat-brain-cortex slices in the presence of $10 \mathrm{~mm}$-glucose. Additions made at $20 \mathrm{~min}$.: $\mathrm{O}$, nil; $\bigcirc$, decanoate; $\triangle, \mathrm{KCl} ; \Delta$, decanoate plus $\mathrm{KCl}$. 
respiratory activity in $45-60 \mathrm{~min}$. Heptanoate produced such an effect at a concentration of 9-10 mM, but dodecanoate, the most effective inhibitor in the series, caused the same effect at a concentration of $0.2 \mathrm{~mm}$. Fatty acids with chain length greater than dodecanoate were found to be relatively less effective.

Effect of washing the slices after treatment with fatty acids. Rat-brain-cortex slices were incubated in the presence of glucose and $0.8 \mathrm{~mm}$-decanoate for various time intervals. They were then removed from the incubation medium, washed with cold Krebs-Ringer solution and incubated in fresh medium containing glucose but no added decanoate. The slices which had been subjected to $20 \mathrm{~min}$. preincubation maintained their respiratory activity in the new medium (Fig. 3). However, slices which had been preincubated with $0.8 \mathrm{~mm}$-decanoate for $60 \mathrm{~min}$. respired at lower rates than did the controls. Thus some form of irreversible damage had occurred.

Effects of decanoate in the presence of various substrates. The effect of decanoate on the respiration of brain-cortex slices in the presence of several carboxylic acids was studied and compared with the effect obtained with glucose as the substrate (Fig. 4). The oxidation of succinate was insensitive to $0.8 \mathrm{~mm}$-decanoate. The respiration in the presence of other substrates, such as fumarate, glutamate, $\alpha$-oxoglutarate and pyruvate, was decreased by decanoate but not to the same extent as was the respiration in the presence of glucose.

\section{Effects of fatty acids on aerobic and anaerobic glycolysis}

A comparison of the effects of potassium decanoate and dinitrophenol (DNP) on respiration, aerobic glycolysis and anaerobic glycolysis in ratbrain-cortex slices is shown in Fig. 5. Decanoate

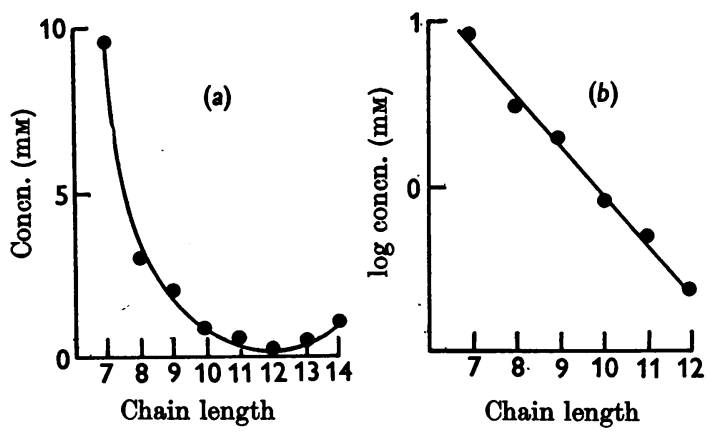

Fig. 2. Relation between chain length of fatty acid and (a) concentration or (b) logarithm of the concentration required to produce a 'fall-off' in respiratory activity of rat-brain-cortex slices in the presence of $10 \mathrm{mM}$-glucose in 45-60 min.

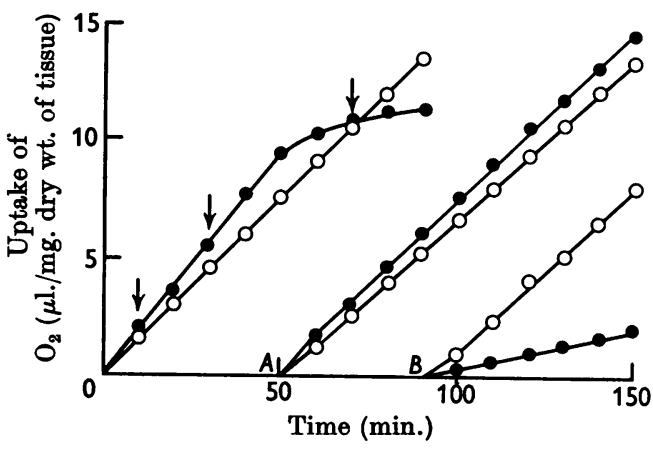

Fig. 3. Effects of washing slices and transferring them to new media after preincubation with $10 \mathrm{~mm}$-glucose and $0.8 \mathrm{~mm}$-potassium decanoate $(O)$ or with $10 \mathrm{~mm}$-glucose only (O). Six Warburg vessels were set up, three with and three without decanoate in the side arms. After incubation for $10 \mathrm{~min}$. (first arrow) decanoate was tipped into the main compartment of the appropriate vessels. After incubation for a further $20 \mathrm{~min}$. one pair of vessels was removed (second arrow); the slices were washed and incubated in fresh media containing $10 \mathrm{~mm}$-glucose but no decanoate. Subsequent respiratory activity is shown in the pair of curves commencing at point $A$. After incubation for a further $40 \mathrm{~min}$., another pair of vessels was removed (third arrow), the slices were washed and incubated as before in fresh media containing $10 \mathrm{~mm}$-glucose but no decanoate. Subsequent respiratory activity is shown in the pair of curves commencing at point $B$. All incubations were carried out at $37^{\circ}$ in a Krebs-Ringer phosphate medium.

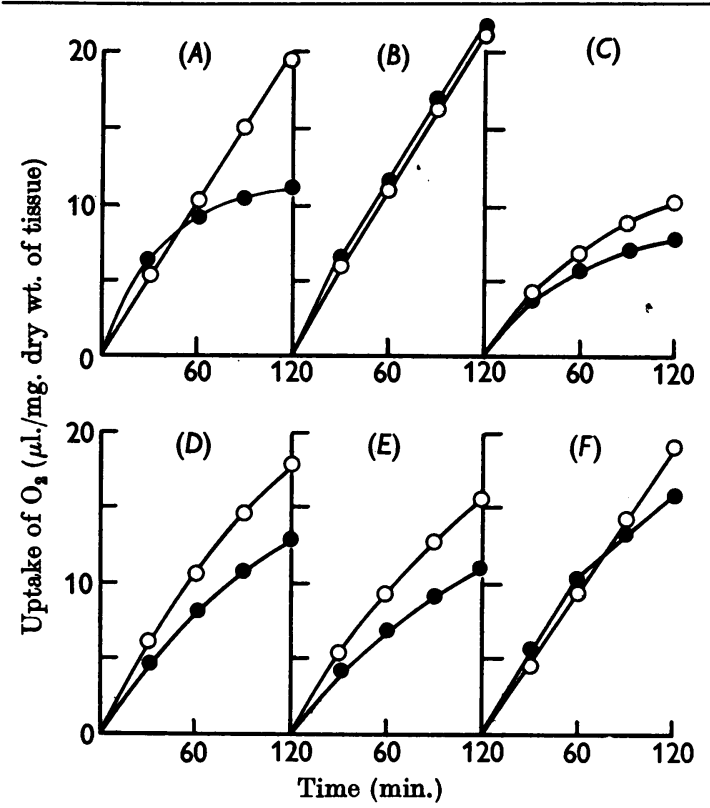

Fig. 4. Effects of $0.8 \mathrm{~mm}$-decanoate on the respiration of rat-brain-cortex slices in the presence of various substrates (each $10 \mathrm{~mm}$ ). Decanoate added: $\bigcirc$, nil; $0,0.8 \mathrm{~mm}$. $(A)$ Glucose; $(B)$ succinate; $(C)$ fumarate; $(D)$ glutamate; $(E) \alpha$-oxoglutarate; (F') pyruvate. 


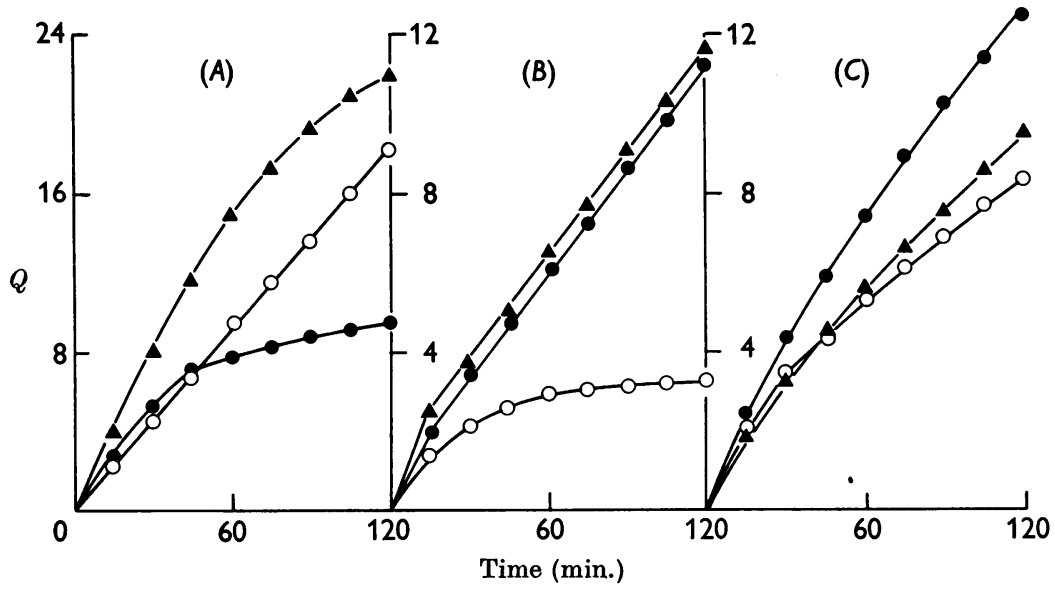

Fig. 5. Effects of $0.8 \mathrm{~mm}$-decanoate and $0.06 \mathrm{~mm}$-DNP on respiration $(A)$, aerobic glycolysis $(B)$ and anaerobic glycolysis $(C)$, by rat-brain-cortex slices. Additions: $O$, nil; $0,0 \cdot 8$ mm-decanoate; $\Delta, 0 \cdot 06$ mM-DNP. Glucose $(10 \mathrm{~mm})$ was present in all vessels. $Q$ values refer in $(A)$ to $\mu$ l. of oxygen uptake, and in $(B)$ and $(C)$ to $\mu$ l. of $\mathrm{CO}_{2}$ evolved/mg. dry wt. of tissue.

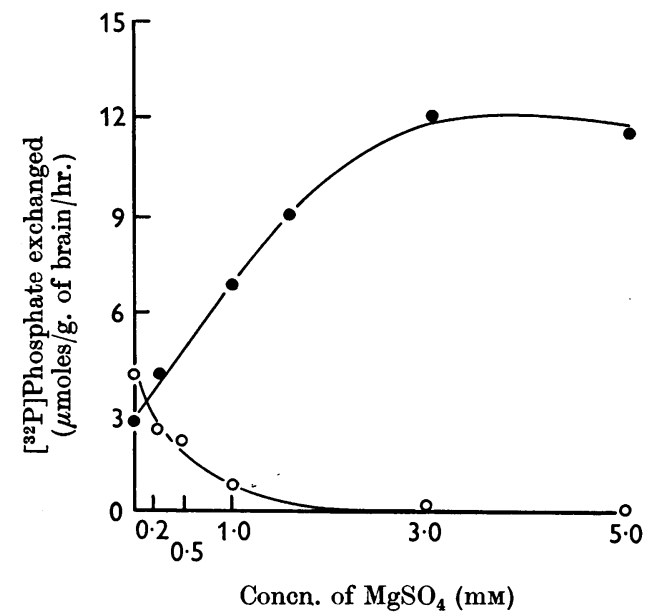

Fig. 6. Effects of $\mathrm{Mg}^{2+}$ ions on the $\mathrm{ATP}-{ }^{32} \mathrm{P}_{i}$ exchange reaction in rat-brain mitochondria in the presence and absence of $10 \mathrm{~mm}$-EDTA. $\bigcirc$, No EDTA present; 0 , 10 mM-EDT'A added.

$(0.8 \mathrm{~mm})$ slightly stimulated anaerobic glycolysis and DNP (0.06 mM) gave a similar result. Aerobic glycolysis in normal slices was low and stopped completely within $60 \mathrm{~min}$. When $0.8 \mathrm{~mm}$-decanoate or $0.06 \mathrm{~mm}$-DNP was added to the incubation medium, the rate of aerobic glycolysis approached that of anaerobic glycolysis.

\section{Effects of decanoate on the adenosine triphosphate- ${ }^{32} \mathrm{P}_{i}$ exchange reaction in rat-brain mitochondria}

The conditions required for the measurement of the $\mathrm{ATP}-{ }^{32} \mathrm{P}_{\boldsymbol{i}}$ exchange in rat-brain mitochondria were found to be somewhat different from those reported for liver mitochondria. When rat-brain mitochondria were isolated from $0.25 \mathrm{M}$-sucrose solution containing $1 \mathrm{~mm}$-ethylenediaminetetraacetic acid (EDTA), they could bring about oxidative phosphorylation at $37^{\circ}$ or $20^{\circ}$, but no exchange reaction could be demonstrated when the incubation was carried out under similar conditions at $20^{\circ}$. This apparent absence of the exchange reaction could not be accounted for by loss of ATP through adenosine-triphosphatase activity since analysis indicated little if any accumulation of inorganic phosphate. Further experiments showed that the occurrence of the ATP_32 $P_{i}$ exchange reaction in these mitochondria could be demonstrated by decreasing the magnesium content of the medium or by adding 10 mM-EDTA (Fig. 6). The complete omission of $\mathrm{Mg}^{2+}$ ions from the medium, however, produced variable results, and experience showed that the most consistent results were obtained when the medium contained both $5 \mathrm{~mm}^{-} \mathrm{MgSO}_{4}$ and $10 \mathrm{~mm}$-EDTA. The labelling of ATP increased linearly with time when mitochondria equivalent to $300 \mathrm{mg}$. original wet wt. of tissue were incubated for $15 \mathrm{~min}$. at $20^{\circ}$. The calculated rate of the ATP-32 $\mathrm{P}_{i}$ exchange reaction in rat-brain mitochondria was $14 \mu$ moles of [ $\left.{ }^{32} \mathrm{P}\right]$ phosphate exchanged/g. wet wt. of brain/hr. The corresponding value found for liver was $84 \mu$ moles (Ahmed \& Scholefield, 1960).

Another major difference between liver and brain mitochondria is their sensitivity towards inhibitors of the exchange reaction, such as DNP and decanoate. DNP (13.5 $\mu \mathrm{M})$ inhibited the exchange reaction in brain by about $75 \%$ (Table 1), whereas in 
Table 1. Effect of decanoate and dinitrophenol on the adenosine triphosphate- ${ }^{32} \mathrm{P}_{\boldsymbol{i}}$ exchange reaction in rat-brain mitochondria

Values in parentheses refer to percentage of the control in each case. The incubation medium contained: ATP, $2.5 \mathrm{~mm} ; \mathrm{MgSO}_{4}, 5 \mathrm{mM} ; \mathrm{KCl}, 37.5 \mathrm{mM}$; tris buffer, $\mathrm{pH} \mathrm{7.4,}$ $100 \mathrm{~mm}$; EDTA, $10 \mathrm{~mm}$; sodium phosphate buffer, $\mathrm{pH} 7 \cdot 4$, $5 \mathrm{~mm}$, and ${ }^{32} \mathrm{P}, 1-2 \mu \mathrm{c}$. Gas phase, air. Mitochondria equivalent to $125 \mathrm{mg}$. wet wt. of brain were added to start the reaction at $20^{\circ}$. Incubation time was $30 \mathrm{~min}$.

$\begin{array}{llc}\text { Inhibitor } & \begin{array}{c}\text { Concn. of } \\ \text { inhibitor } \\ (\mathrm{mm})\end{array} & \begin{array}{c}\text { Phosphate } \\ \text { exchanged } \\ (\mu \text { moles/hr./g. } \\ \text { wet wt. of tissue) }\end{array} \\ \text { Decanoate } & 0 & 14 \cdot 0(100) \\ & 0 \cdot 1 & 14 \cdot 2(102) \\ & 0 \cdot 2 & 12 \cdot 3(88) \\ & 0 \cdot 3 & 8 \cdot 9(64) \\ \text { DNP } & 0 \cdot 4 & 7 \cdot 8(56) \\ & 0 & 13 \cdot 4(100) \\ & 0 \cdot 0045 & 9 \cdot 3(69) \\ & 0 \cdot 009 & 6 \cdot 9(52) \\ & 0.0135 & 3 \cdot 6(27)\end{array}$

liver mitochondria a comparable inhibition of the exchange may be observed in the presence of $5 \mu \mathrm{M}$ DNP. No significant increase in the rate of the ATP $-{ }^{32} P_{i}$ exchange reaction in brain mitochondria could be observed in the presence of very low concentrations of decanoate such as occurs in liver mitochondria (Ahmed \& Scholefield, 1960). The concentration of decanoate required to produce a $50 \%$ inhibition of the exchange reaction, being about $0.4 \mathrm{mM}$, is of the same order as that required to produce a $50 \%$ inhibition of oxidative phosphorylation in brain mitochondria (Scholefield, 1956).

\section{Effects of decanoate on incorporation of ${ }^{32} \mathrm{P}$ into brain-cortex slices}

Time course of ${ }^{32} \mathrm{P}$ incorporation. The labelling of organic phosphate compounds with ${ }^{32} \mathrm{P}$ in brain slices with glucose as substrate takes place very slowly. In order to increase the intensity of labelling, the specific activity of the phosphate was increased by reducing the concentration of carrier inorganic phosphate in the incubation medium from 10 to $3.3 \mathrm{~mm}$. The uptake of phosphate from the medium containing $3.3 \mathrm{~mm}$-phosphate was about one-third of that obtained in the presence of 10 mM-phosphate (Table 2). The labelling of this fraction and of the organic phosphates continued to increase during an incubation period of $120 \mathrm{~min}$. The difference in the amount of carrier phosphate did not produce a significant effect on the rate of phosphate incorporation into various organic phosphate compounds, when the latter were expressed in terms of $\mu \mathrm{m}$-moles of phosphate $/ 100 \mathrm{mg}$. of tissue, with the possible exception of the phospholipid and acid-insoluble fractions.

Effects of decanoate on incorporation of ${ }^{32} \mathrm{P}$ with added glucose. The effects of 0.3 and $0.6 \mathrm{~mm}$ decanoate on incorporation of ${ }^{32} \mathrm{P}$ into rat-braincortex slices in the presence of glucose were studied. Decanoate $(0.3 \mathrm{~mm})$ slightly stimulated respiration during the entire incubation period whereas $0.6 \mathrm{~mm}$ decanoate began to inhibit respiration after incubation for $60 \mathrm{~min}$. No effect on the uptake of inorganic phosphate was observed (Table 3) but there was a marked effect on the phosphate incorporation into the organic phosphates. Decanoate $(0.3 \mathrm{~mm})$ inhibited incorporation of ${ }^{32} \mathrm{P}$ into the phospholipid, ATP plus ADP, acid-insoluble and 'other nucleotides' fractions by about $50 \%$ when the slices were incubated for $30 \mathrm{~min}$., the inhibition being increased to about $80 \%$ at the end of incubation for $120 \mathrm{~min}$. With $0.6 \mathrm{~mm}$-decanoate, the incorporation of ${ }^{32} \mathrm{P}$ of all the fractions was inhibited by more than $80 \%$ within $30 \mathrm{~min}$. Subsequently the rate of oxygen uptake began to decrease rapidly and there was little respiration in the presence of $0.6 \mathrm{~mm}$-decanoate during the second hour of incubation. The level of radioactivity in the inorganic phosphate fraction rose but the levels in all the organic phosphate fractions fell.

Incorporation of ${ }^{32} \mathrm{P}$ into brain-cortex slices in the presence of other substrates. Incorporation of ${ }^{32} \mathrm{P}$ into various fractions was studied with glucose, succinate, glutamate, pyruvate and $\alpha$-oxoglutarate as substrates (Table 4). The presence or absence of substrate appeared to have relatively little effect on the uptake of phosphate. The incorporation of phosphate, however, greatly depended upon the nature of the substrate added. Maximum incorporation of phosphate into the various organic phosphate fractions took place only in the presence of glucose and was minimal when no substrate was added. With succinate or glutamate as substrate, the phosphate incorporation into all the organic phosphate fractions, including the ATP plus ADP fraction, was of the same order as when no substrate was added. Phosphate incorporation with pyruvate or $\alpha$-oxoglutarate as substrate was slightly greater than with the other substrates but was still only about $25-50 \%$ of the values obtained with glucose [see values for ATP plus ADP, phospholipids etc. (Table 4)]. The labelling of ATP, ADP and the other nucleotides was maximal only when glucose was used as substrate.

The effects of $0.6 \mathrm{~mm}$-decanoate and $0.05 \mathrm{~mm}$ DNP on incorporation of [ $\left.{ }^{32} \mathrm{P}\right]$ phosphate into brain slices with succinate as substrate were also studied. As shown above, the phosphate incorporation in the presence of this substrate is very low and these inhibitors reduced it to still lower values.

Bioch. 1961, 81 
Table 2. Time course of incorporation of $\left[{ }^{32} \mathrm{P}\right]$ phosphate into various fractions in rat-brain-cortex slices

All values refer to $\mu \mathrm{m}$-moles of phosphate incorporated/100 mg. wet wt. of brain-cortex slices. Other nucleotides included UTP, GTP and GDP. Slices were incubated at $37^{\circ}$ in Krebs-Ringer medium containing the amounts of phosphate buffer indicated. Glucose $(10 \mathrm{mM})$ was present as substrate and the gas phase was $\mathrm{O}_{2}$.

Concn. of

phosphate, Incubation

$\mathrm{pH} \mathbf{7 \cdot 4}$

(mM)

time

10

30

120

$\begin{array}{rc} & \text { ATP plus } \\ \mathbf{P}_{\boldsymbol{i}} & \text { ADP } \\ 1020 & 28 \\ \mathbf{2 7 9 0} & \mathbf{5 3} \\ \mathbf{3 6 0} & 29 \\ \mathbf{7 2 0} & \mathbf{6 0}\end{array}$

Other
nucleotides
9
22
6
18

Phospholipids
11
31
10
26

Acid-insoluble fraction

$3 \cdot 3$

120

Table 3. Effect of decanoate on incorporation of $\left[{ }^{32} \mathrm{P}\right]$ phosphate into rat-brain-cortex slices

All values refer to $\mu \mathrm{m}$-moles of phosphate incorporated/100 mg. wet wt. of brain-cortex slices. All other conditions were the same as for Table 2, the concentration of phosphate being $\mathbf{3 \cdot 3} \mathrm{mm}$.

Incubation
time
(min.)

30

120

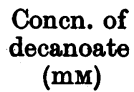

Nil

$0 \cdot 3$

0.6

Nil

$0 \cdot 3$

0.6

$\mathbf{P}_{\boldsymbol{i}}$
261
207
250
443
363
325

ATP plus
ADP
23
14
2
63
10
1

Acid-

insoluble

Phospholipids

fraction

$\begin{array}{rr}7 \cdot 9 & 6 \\ 2 \cdot 7 & 4 \\ 1.5 & 1 \\ 15.4 & 16 \\ 4 \cdot 8 & 8 \\ 0.8 & 1\end{array}$

Table 4. Effect of various substrates on incorporation of $\left[{ }^{32} \mathrm{P}\right]$ phosphate into rat-brain-cortex slices

All values refer to $\mu \mathrm{m}$-moles of phosphate incorporated/100 mg. wet wt. of rat-brain-cortex slices. Incubation was for 60 min. at $37^{\circ}$ in a Krebs-Ringer medium containing 3.3 mM-phosphate buffer, $\mathrm{pH} \mathrm{7 \cdot 4}$.

\begin{tabular}{|c|c|c|c|c|c|c|}
\hline $\begin{array}{c}\text { Substrate } \\
(10 \mathrm{~mm})\end{array}$ & $\begin{array}{c}\text { Total } \\
\text { acid-soluble } \\
\text { phosphates }\end{array}$ & $\mathbf{P}_{\boldsymbol{i}}$ & Phospholipids & $\begin{array}{c}\text { Acid- } \\
\text { insoluble } \\
\text { fraction }\end{array}$ & $\begin{array}{l}\text { ATP plus } \\
\text { ADP }\end{array}$ & $\begin{array}{c}\text { Other } \\
\text { nucleotides }\end{array}$ \\
\hline $\begin{array}{l}\text { Nil } \\
\text { Glucose } \\
\text { Succinate } \\
\text { Glutamate } \\
\text { Pyruvate } \\
\alpha \text {-Oxoglutarate }\end{array}$ & $\begin{array}{l}\mathbf{3 7 6} \\
\mathbf{3 3 2} \\
\mathbf{3 7 3} \\
\mathbf{3 4 3} \\
\mathbf{3 8 9} \\
\mathbf{3 8 8}\end{array}$ & $\begin{array}{l}345 \\
292 \\
\mathbf{3 6 0} \\
\mathbf{3 3 3} \\
\mathbf{3 7 2} \\
\mathbf{3 6 9}\end{array}$ & $\begin{array}{l}3 \cdot 4 \\
9 \cdot 0 \\
2 \cdot 6 \\
2 \cdot 5 \\
4 \cdot 3 \\
3 \cdot 8\end{array}$ & $\begin{array}{r}4 \\
12 \\
4 \\
5 \\
8 \\
6\end{array}$ & $\begin{array}{r}6 \\
41 \\
6 \\
5 \\
12 \\
13\end{array}$ & $\begin{array}{l}2 \cdot 5 \\
4 \cdot 9 \\
2 \cdot 5 \\
2 \cdot 2 \\
3 \cdot 9 \\
3 \cdot 4\end{array}$ \\
\hline
\end{tabular}

\section{DISCUSSION}

Decanoate and other fatty acids (between $\mathrm{C}_{7}$ and $\mathrm{C}_{14}$ ) cause a gradual cessation of respiratory activity of brain-cortex slices incubated with glucose. The phenomenon appears to be characteristic of this tissue and was not observed with either kidney or liver slices, where inhibition of respiratory activity of tissues increases much more slowly with time. This 'fall-off' of respiration may be correlated with the effects of decanoate and DNP on glycolysis. Anaerobic glycolysis of brain slices is only slightly stimulated by these agents but the effect on aerobic glycolysis is very striking. In the absence of decanoate or DNP, brain-cortex slices exhibited a low aerobic glycolysis, which ceased completely within $45 \mathrm{~min}$. In the presence of $0.8 \mathrm{~mm}$-decanoate or $0.06 \mathrm{~mm}-\mathrm{DNP}$ the aerobic glycolysis of rat- brain-cortex slices was greatly stimulated and remained at a high rate for more than $120 \mathrm{~min}$. It is well known that uncoupling agents can reverse the Pasteur effect (Dickens, 1951) and the fact that decanoate behaves in a similar fashion is undoubtedly a consequence of its ability to act as an uncoupling agent. A stimulation of aerobic glycolysis, when slices are respiring with glucose as substrate, yields a large amount of lactic acid and a sufficient amount may accumulate inside the cell to cause a lowering of the internal $\mathrm{pH}$. This would result in cellular damage and produce the 'fall-off' of respiratory activity similar to that observed in Ehrlich ascites-carcinoma cells (Scholefield, 1958). Further evidence to favour this suggestion was obtained from experiments in which slices were incubated with glucose in the presence or absence of decanoate for different periods of time 
and then transferred to fresh media. The inhibition of respiration resulting from the presence of decanoate was irreversible. The preliminary incubation had thus produced permanent damage, which, in this case, was probably due to $\mathrm{pH}$ effects. Further, this 'fall-off' is observed only in the presence of glucose and did not occur when other substrates, which do not give rise to large amounts of acids such as lactic acid, were used. The inhibitory effects increased to a maximum with chain length $\mathrm{C}_{12}$, the fatty acids having a greater number of carbon atoms being less effective. The higher fatty acids, with their greater lipid solubility, may tend to be retarded by the cell membrane and so be less effective than the fatty acids of medium chain length.

The effects of decanoate on incorporation of ${ }^{32} \mathrm{P}$ into brain-cortex slices lend support to the suggestion that the membrane may play a role in determining the effectiveness of the fatty acids. Thus 0.3 mM-decanoate had little effect on brain respiration during the course of an incubation period of $2 \mathrm{hr}$. However, it gave rise to about $50 \%$ inhibition of the labelling of the ATP plus ADP fraction of this tissue during the first $30 \mathrm{~min}$. of incubation and completely inhibited further incorporation in the next $90 \mathrm{~min}$. The ability of decanoate to alter the rate of incorporation of ${ }^{32} \mathrm{P}$ into slices of rat-brain cortex thus increases with the time of incubation, which suggests that a limited rate of penetration of the slice by the fatty acid may be involved.

In isolated rat-brain mitochondria the concentration of decanoate required to uncouple oxidation from phosphorylation by $50 \%$ is $0.6 \mathrm{~mm}$ (Scholefield, 1956). The sensitivity of rat-brain slices to a lower concentration of decanoate $(0.3 \mathrm{mM})$, even in the first $30 \mathrm{~min}$. of incubation, suggests that an effect of the membrane of the brain cells may be involved. Alternatively, the ATP $-{ }^{32} P_{i}$ exchange reaction may account for much of the labelling of ATP with ${ }^{32} \mathrm{P}$ in slices, as has previously been shown to occur in liver (Ahmed \& Scholefield, 1960). The results shown in Table 1 indicate that the exchange reaction in brain mitochondria is about as sensitive as is the process of oxidative phosphorylation, so that there is no reason to suppose that an exchange reaction is primarily responsible for the labelling with ${ }^{32} \mathrm{P}$ in brain slices. Indeed, the slow decline in the rate of labelling of the ATP in a cortex slice over the incubation period of $2 \mathrm{hr}$. and the failure to attain a steady-state level in this time would indicate otherwise. In liver, where the exchange reaction does take place rapidly in the mitochondria, the ATP of slices acquires a steady-state level of radioactivity within $20 \mathrm{~min}$. (Ahmed \& Scholefield, 1961). On the other hand, the observed rate of labelling in rat-brain-cortex slices in the absence of added inhibitor is much slower than would have been expected. Assuming a $Q_{\mathrm{O}_{2}}$ of 10 and a $\mathrm{P} / \mathrm{O}$ ratio of 2.5 there should be approximately $45 \mu$ moles of $P_{i}$ incorporated into $\mathrm{ATP} / \mathrm{hr} . / 100 \mathrm{mg}$. wet wt. of brain, and the observed average rate for the first $30 \mathrm{~min}$. of incubation, based on incorporation of ${ }^{32} \mathrm{P}$, is $45 \mu \mathrm{m}-\mathrm{moles} / \mathrm{hr}$. or $0.1 \%$ of the theoretical rate. It seems therefore that some of the membranes restricting the interchange of phosphate between the inorganic form and the ATP may well be intracellular.

Difficulties were at first encountered in assaying the ATP- ${ }^{32} \mathrm{P}_{i}$ exchange reaction in brain mitochondria. Media which support oxidative phosphorylation in liver also support the exchange reaction (Cooper \& Lehninger, 1957). This is not so in brain, where the medium used to measure $\mathrm{P} / \mathrm{O}$ ratios at $20^{\circ}$ or $37^{\circ}$ permitted only slow incorporation of ${ }^{32} \mathrm{P}$ into ATP by the exchange reaction at $20^{\circ}$. In the presence of protein $(0.67 \%$ of bovine serum albumin) better exchange could be detected but the best results were obtained on addition of 10 mM-EDTA. A variable increase could also be detected on omission of $\mathrm{Mg}^{2+}$ ions from the incubation medium, but the most consistent results were obtained when both $10 \mathrm{~mm}$-EDTA and $5 \mathrm{~mm}-\mathrm{Mg}^{2+}$ ions were present. EDTA, besides removing factors which may be inhibitory to the exchange reaction, may also be chelating a considerable quantity of $\mathrm{Mg}^{2+}$ ions and maintaining a very low but critical concentration of these ions. This would account for the inhibition of the exchange reaction in brain by excess of $\mathrm{Mg}^{2+}$ ions in the presence of $10 \mathrm{~mm}$ EDTA (Fig. 6).

Another factor leading to the conclusion that the labelling of the organic phosphates of brain slices with ${ }^{32} \mathrm{P}$ is due to net synthesis is the specificity of the substrate supporting the respiration. Of the substrates examined, only glucose brought about a high rate of phosphate incorporation, a rate of approximately six times the value observed in the absence of added substrate being obtained. Pyruvate and $\alpha$-oxoglutarate slightly increased the phosphate incorporation whereas succinate and glutamate failed to raise the level above that of the endogenous, although the respiratory activity of the slices was high. Earlier studies on phosphate incorporation into brain slices showed that glutamate and succinate, though increasing the oxygen consumption of the slices, failed to support the incorporation of [ $\left.{ }^{32} \mathrm{P}\right]$ phosphate into phospholipids, phosphoproteins, nucleic acids and residue organic phosphorus fractions (Findlay et al. 1953; Strickland, 1954). It is now shown that in the presence of these substrates the incorporation of [ $\left.{ }^{32} \mathrm{P}\right]$ phosphate into ATP is as low as it is in the other fractions. Thus the low rate of incorporation of ${ }^{32} \mathrm{P}$ into the 
other organic phosphates is probably a consequence of a low ${ }^{32} \mathrm{P}$ turnover in the ATP plus ADP fraction.

Weil-Malherbe (1950) has pointed out that glutamate may not be a major energy-yielding substrate in brain. A number of observations support this conclusion (see, for example, McIlwain, 1951 $a$, $b, 1953$; McIlwain \& Gore, 1953; Waelsch, 1951). Similar considerations may apply to succinate since Kratzing (1953) has shown that guinea-pigbrain slices respiring in succinate did not respond to electrical stimulations and also failed to maintain their levels of phosphocreatine and ATP. Quastel, Tennenbaum \& Wheatley (1936) had already shown that optimum synthesis of acetylcholine in brain slices takes place only in the presence of glucose and oxygen. The synthesis was not supported by succinate and only partially supported by pyruvate or glutamate. Similarly, Stern, Eggleston, Hems \& Krebs (1949), who studied the accumulation of glutamate in slices against a concentration gradient, found that of the various additional substrates studied only glucose supported the maximum rate of accumulation of glutamate. On the other hand, Abood \& Gerard (1952) and Brody \& Bain (1952) have shown that brain mitochondria may yield $\mathrm{P} / \mathrm{O}$ ratios approaching the theroretical values during the oxidation of succinate, glutamate or other substrates. Similarly, labelling of phospholipids with ${ }^{32} \mathrm{P}$ in rat-brain mitochondria has been demonstrated in the presence of these substrates (McMurray, Berry \& Rossiter, 1957). The oxidation of substrates such as succinate and glutamate by slices may not be properly coupled to phosphorylation or the ATP so formed may not be available for the synthetic processes or to the cellular pool of radioactive phosphate.

The rate of incorporation of ${ }^{32} \mathrm{P}$ into braincortex slices is much lower under anaerobic conditions than it is under aerobic conditions (Findlay et al. 1953; Strickland, 1954). In the present studies, incorporation of ${ }^{32} \mathrm{P}$ into the ATP plus ADP fraction under anaerobic conditions was about $5 \%$ of that observed under aerobic conditions. Low incorporation of ${ }^{32} \mathrm{P}$ into the ATP plus ADP fraction under anaerobic conditions might have been expected. The relative yields of ATP may be expressed (Quastel \& Bickis, 1959) by the ratio $Q_{\mathrm{CO}_{3}}^{\mathrm{N}_{3}} / 6 \times Q_{\mathrm{O}_{3}}$. The observed values for $Q_{\mathrm{CO}_{2}}^{\mathrm{N}_{2}}$ and $Q_{0}$, were 7 and 11 respectively, so that the ratio is $7 / 66$ and the yield of ATP under anaerobic conditions would be expected to be about $10 \%$ of that to be obtained under aerobic conditions. This is of the same order as the figure of $5 \%$ quoted above for the ratio of the rates of labelling of ATP with ${ }^{32} \mathrm{P}$ and is in contrast with results from tumours, where it has been shown (Quastel \& Bickis, 1959) that the $Q_{\mathrm{ATP}}$ in the presence of glucose is independent of the presence of oxygen.

\section{SUMMARY}

1. Decanoate and other fatty acids initially stimulated the respiratory activity of rat-braincortex slices incubated in Krebs-Ringer glucose medium. Subsequently the respiratory activity ceased gradually and irreversibly. The time interval between these two effects depended upon the concentration and chain length of the acid.

2. Of the fatty acids from heptanoate to myristate, heptanoate was the least effective as an inhibitor and decanoate was the most effective. Saturated fatty acids of greater chain length were less effective. Decanoate $(0.8 \mathrm{~mm})$ brought about an inhibition of respiration of rat-brain-cortex slices after an incubation period of 45-60 min.

3. The greatest inhibitory effects of decanoate were obtained when glucose was present as the substrate. When the acids of the carboxylic acid cycle were used as substrates the respiration was not inhibited to the same extent as with glucose, nor was the inhibition complete.

4. Decanoate and dinitrophenol greatly stimulated aerobic glycolysis at concentrations which were inhibitory to respiration. Anaerobic glycolysis was stimulated slightly by these agents.

5. Decanoate inhibited phosphate incorporation into all the organic phosphate compounds at concentrations which did not significantly alter the respiratory activity of rat-brain-cortex slices.

6. The adenosine triphosphate ${ }^{-32} \mathrm{P}_{i}$ exchange reaction in rat-brain mitochondria is not as rapid as it is in liver mitochondria. It is best measured in the presence of $10 \mathrm{~mm}$-ethylenediaminetetra-acetate and at least $3 \mathrm{~mm}-\mathrm{Mg}^{2+}$ ions. The inhibitory effects of decanoate on this exchange reaction and on coupled phosphorylation are quantitatively similar.

It is a pleasure to thank Professor J. H. Quastel, F.R.S., for his continued interest in this work and to acknowledge the financial support of the National Cancer Institute of Canada. One of us (K.A.) was the holder of a Colombo Plan Fellowship under the Technical Assistance Programme by the Government of Canada, Department of Trade and Commerce, to the Republic of Pakistan.

\section{REFERENCES}

Abood, L. G. \& Gerard, R. W. (1952). Amer. J. Physiol. 168, 739 .

Ahmed, K. \& Scholefield, P. G. (1960). Nature, Lond., 186, 1046.

Ahmed, K. \& Scholefield, P. G. (1961). Biochem. J. 81, 37. Brody, T. M. \& Bain, J. A. (1952). J. biol. Chem. 195, 685. Cooper, C. \& Lehninger, A. L. (1957). J. biol. Chem. 224, 547.

Dickens, F. (1951). In Enzymes, vol. 2, part 1, p. 624. Ed. by Sumner, J. B. \& Myrbäck, K. New York: Academic Press Inc. 
Findlay, M., Rossiter, R.' J. \& Strickland, K. P. (1953). Biochem. J. 55, 200.

Kratzing, C. C. (1953). Biochem. J. 54, 312.

McIlwain, H. (1951a). J. ment. Sci. 97, 674.

Mcllwain, H. (1951 b). Biochem. J. 49, 382; 50, 12.

Mcllwain, H. (1953). J. Neurol. Psychiat. 16, 257.

McIlwain, H. \& Gore, M. B. R. (1953). Biochem. J. 54, 305.

McMurray, W. C., Berry, J. F. \& Rossiter, R. J. (1957). Biochem. J. 66, 629.

Quastel, J. H. \& Bickis, I. J. (1959). Nature, Lond., 183, 281.

Biochem. J. (1961) 81, 53
Quastel, J. H., Tennenbaum, M. \& Wheatley, A. H. M. (1936). Biochem. J. 80, 1668.

Samson, F. E. \& Dahl, N. (1955). Fed. Proc. 14, 129.

Scholefield, P. G. (1956). Canad. J. Biochem. Physiol. 34, 1211.

Scholefield, P. G. (1958). Cancer Res. 18, 1026.

Stern, J. R., Eggleston, L. V., Hems, R. \& Krebs, H. A. (1949). Biochem. J. 44, 410.

Strickland, K. P. (1954). Canad. J. Biochem. Physiol. 32, 50.

Waelsch, H. (1951). Advanc. Protein Ohem. 6, 299.

Weil-Malherbe, H. (1950). Physiol. Rev. 30, 549.

\title{
Further Studies on the Absorption of Vitamin A
}

\author{
By S. MAHADEVAN AND J. GANGULY \\ Department of Biochemistry, Indian Institute of Science, Bangalore 12, India
}

\section{(Received 2 February 1961)}

Irrespective of whether vitamin A is fed to rats as the free alcohol or as its esters, $75 \%$ of it is found in the esterified form in the mucosae and muscles of the small intestine, whereas only the ester is transported exclusively through the lymphatic system, so that in the post-absorptive blood the ester shows a sharp increase (Ganguly, 1960). Gray \& Cawley (1942) concluded from the molecular-distillation data on the oil obtained from rat liver that vitamin A was probably stored in the liver as its palmitate. Later work of Kaiser \& Kagan (1956) showed that the vitamin A ester of blood and liver of rats was composed of higher fatty acids, and Mahadevan, Krishnamurthy \& Ganguly (1959) showed that the lower esters of vitamin A were not absorbed from the intestine.

Gray, Hickman \& Brown (1940) suggested that when rats are fed with different types of esters of vitamin A the liver, in time, converts them into one type of ester, probably the palmitate. We have recently demonstrated that an enzyme preparation from the intestinal mucosae of rats can rapidly esterify vitamin A with almost equal efficiency with saturated or unsaturated fatty acids containing more than 10 carbon atoms. However, it fails to esterify the vitamin with acids containing fewer carbon atoms (Murthy, Mahadevan, Seshadri Sastry \& Ganguly, 1961). It might therefore be assumed that different types of esters (depending upon the availability of fatty acids of either endogenous or exogenous source) would be present in the mucosae and muscles of the small intestine, as well as in the blood and liver of rats, after a dose of the vitamin. It is demonstrated here that contrary to this expectation, it is the palmitate of vitamin A that always predominates in the mucosae, muscles and blood, and only the palmitate is deposited in the liver of rats.

\section{MATERIALS AND METHODS}

Methanol (Merck; Pro Analyst grade) and butanol (British Drug Houses Ltd.; Laboratory Reagent grade) were redistilled before use. Tweens 20, 40 and 60 (lauric, palmitic and stearic acid esters respectively of polyoxyethylene sorbitol) were obtained from Atlas Powder Co., Wilmington, Del., U.S.A. Refined coconut oil was obtained from the local market. Groundnut, sesame (Sesamum indicum) and safflower (Carthamus tinctorius) oils were expressed in an expeller from the respective oil seeds commercially available and were used without further purification. Silicone fluid F 110/300 was from Imperial Chemical Industries Ltd., London. The rest of the materials have been described in previous papers (Mahadevan et al. 1959; Ganguly, Krishnamurthy \& Mahadevan, 1959).

Synthetic vitamin A alcohol, purified by chromatography on alumina columns (Ganguly, Krinsky, Mehl \& Deuel, 1952), was dissolved in light petroleum (b.p. 40-60 together with the Tween or the oil so as to obtain a final concentration of $6 \mathrm{mg}$. of the vitamin/ml. of the carrier. The solvent was evaporated under vacuum at $40-50^{\circ}$, the last traces being removed with a gentle stream of $\mathrm{N}_{2}$. Colloidal dispersions were prepared according to Mahadevan et al. (1959).

Rats of either sex of this Institute strain were maintained after weaning on a vitamin-A-low diet composed of (\%): starch, 65; solvent-extracted casein, 20; groundnut oil, 10; salt mixture, 5; with daily supplements of adequate amounts of the other vitamins. The animals were used after they had been on the diet for 5-6 weeks; at this stage their livers were essentially free from vitamin $A$. 